\title{
Sentimientos y teoría de la acción
}

\author{
CARLOS J. MOYA
}

Universidad de Valencia

Resumen. En el presente trabajo sostenemos que la concepción de la intencionalidad en la teoría de la acción más ampliamente aceptada en la actualidad hace difícil una comprensión adecuada del papel de las emociones en la génesis e interpretación de la acción. La asimilación de las emociones a actitudes intencionales descuida lo que cabría llamar su contenido emocional y pasa por alto importantes diferencias entre su contenido intencional y el de las actitudes intencionales paradigmáticas, como creencias, deseos e intenciones. Sugerimos, sobre esta base, que las relaciones externas y causales deberían tener un lugar más destacado en la comprensión y explicación de las acciones humanas.
Abstract. In this paper it is held that the view of intentionality in the most influential action theory nowadays makes it difficult to adequately understand the role of emotions in the origin and interpretation of action. Assimilating emotions to intentional attitudes neglects what might be called the emotional content of the former and loses sight of important differences in intentional content between emotions and paradigmatic intentional attitudes, such as beliefs, desires, and intentions. On this basis, it is suggested that external and causal relations should play a more distinguished role in understanding and explaining human actions.

La teoría de la acción intencional más ampliamente aceptada en la actualidad, que cabría llamar el modelo creencia/deseo, uno de cuyos principales defensores es Donald Davidson, no concede a las emociones y sentimientos un papel explícito en la explicación de la acción. La razón es que, en este marco, las emociones son asimiladas a creencias o deseos, o a combinaciones de ambas, o bien son consideradas como actitudes intencionales peculiares. Sostendremos que esta visión de las emociones deriva de la concepción wittgensteiniana de la intencionalidad, que influye en Davidson a través de pensadoras como Elizabeth Anscombe. Esta concepción, sin embargo, margina aspectos muy importantes de determinados estados mentales, entre los que se encuentra lo que cabría llamar su contenido emocional. Por otra parte, el contenido intencional de las emociones presenta importantes diferencias con respecto al contenido intencional de las actitudes intencionales paradigmáticas, como la creencia, el deseo o la intención, por lo que resulta confundente la asimilación o reducción de aquéllas a éstas. La atención al contenido emocional y a las peculiaridades del contenido intencional de las emociones aconseja un replanteamiento en profundidad del modelo creencia/deseo, cuyas líneas generales son apuntadas al final de este trabajo. 


\section{El modelo creencia/deseo de la explicación de la acción}

La comprensión filosófica de la acción en los últimos treinta años ha estado fuertemente dominada por el análisis davidsoniano de la acción intencional. Una acción, para Davidson, es un episodio de conducta que, bajo alguna descripción verdadera, es intencional. Y una acción es intencional, bajo una determinada descripción, sólo si, así descrita, posee una explicación verdadera en términos de razones. Una explicación así requiere al menos dos condiciones.

En primer lugar, la razón ha ser una razón primaria, lo que significa que ha de estar constituida, al menos implícitamente, por una actitud favorable (cuyo paradigma es el deseo) del agente hacia acciones de cierto tipo en la medida en que posean cierta propiedad y por una creencia según la cual una determinada acción, bajo una determinada descripción, posee dicha propiedad (o, lo que es lo mismo, pertenece a ese tipo) ${ }^{1}$. La precisión «en la medida en que» es fundamental para dar cuenta del hecho obvio de que no llevamos a cabo todas aquellas acciones para las que tenemos razones, puesto que es perfectamente compatible tener una actitud favorable hacia una acción en la medida en que tiene cierta propiedad y una actitud desfavorable o contraria a ella en la medida en que tiene otras propiedades. Así, del hecho de que un agente posea una razón con esa estructura para cierta acción no se sigue la realización de dicha acción, sino sólo un juicio sobre la deseabilidad de la acción relativo a ciertas consideraciones (en particular, que la acción tiene la propiedad en cuestión).

Que la razón posea esta estructura interna es fundamental para que pueda desempeñar su papel característico de justificación racional de la acción. Juzgamos razonable que un agente actúe de cierta forma cuando vemos esa acción como un intento del agente de satisfacer su deseo de ejemplificar la propiedad en cuestión a la luz de su creencia de que esa acción ejemplifica dicha propiedad, o, en términos más coloquiales, cuando vemos la acción como un medio que el agente cree o juzga adecuado para satisfacer su deseo.

En segundo lugar $-\mathrm{y}$ éste es el requisito que hace de la teoría davidsoniana de la acción una teoría causal- para que una razón con la estructura interna indicada pueda explicar una acción, es necesario también que dicha razón sea la razón por la cual el agente lleva a cabo la acción. Un agente puede

'Esta concepción de una razón primaria se aparta del análisis desarrollado por Davidson en su célebre artículo «Acciones, razones y causas», y toma en cuenta elementos de trabajos posteriores, en especial «How is Weakness of the Will Possible» e «Intending». Estos trabajos se encuentran recogidos en D. Davidson, Essays on Actions and Events, Oxford, Clarendon Press, 1982 (hay traducción castellana en Barcelona, Crítica, 1995). La modificación consiste fundamentalmente en la sustitución de la frase «acciones de cierto tipo que tengan cierta propiedad» por «acciones de cierto tipo en la medida en que tengan cierta propiedad». Esta sustitución, en apariencia poco significativa, resuelve, sin embargo, problemas muy importantes. Sobre esta cuestión véase mi libro The Philosophy of Action, Cambridge, Polity Press, 1990, pp. 145-148. 
llevar a cabo una acción para la que tiene una razón con la estructura interna adecuada y, sin embargo, llevar a cabo la acción por otra razón distinta. En este caso, sólo la última razón, y no la primera, explica la realización de la acción. Y el modo más natural de especificar ese requisito y distinguir ambos casos es considerar la razón por la cual el agente actúa como causa de su acción.

Ambas condiciones son necesarias para que una razón explique una acción y ésta sea, bajo la descripción adecuada, intencional, pero, desgraciadamente, no son conjuntamente suficientes, debido a que el modo en que la razón causa la acción puede ser inapropiado o «desviado». Habría, pues, que añadir una especificación del modo en que una razón, con la estructura interna adecuada, ha de causar una acción para obtener condiciones no sólo necesarias, sino también suficientes, de la acción intencional. Sin embargo, Davidson no cree que sea posible llevar a cabo dicha especificación de una manera que no sea circular, es decir, que no presuponga ya el concepto mismo de acción intencional que se pretende analizar ${ }^{2}$. Este problema, conocido en la bibliografía filosófica como el problema de las cadenas causales desviadas, constituye una debilidad endémica de las teorías causales de la acción, davidsonianas o no ${ }^{3}$. Sin embargo, a efectos de nuestra argumentación, podemos aceptar una solución nominal (y seguramente circular) a este problema asumiendo, como es habitual en la bibliografía, que las razones han de causar la acción del modo apropiado. Cabe decir entonces (siendo conscientes de que el problema de la desviación causal espera todavía una solución efectiva) que una acción es intencional, bajo una determinada descripción, si, y sólo si, bajo esa descripción, tiene una explicación verdadera en términos de razones.

La concepción de la acción intencional en términos de su explicación característica mediante razones que acabamos de esbozar requeriría importantes refinamientos para dar cuenta de casos problemáticos de conflicto de deseos y/o creencias y otros. Sin embargo, en su estructura básica, que confiamos haber expuesto en lo sustancial, esta concepción, conocida a veces como «el modelo creencia/deseo» de la explicación de la acción, informa decisivamente la reflexión filosófica y empírica actual sobre la acción racional, incluyendo la teoría de la decisión y zonas importantes de la llamada concepción cognitiva de la mente y de la acción.

${ }^{2}$ Así, en «Freedom to Act», Davidson escribe: «De lo que desespero es de especificar el modo en que las actitudes han de causar acciones para que puedan explicarlas racionalmente» (Essays on Actions..., p. 79).

${ }^{3}$ Al respecto puede verse el capítulo 11 de mi The Philosophy of Action. Un intento de diagnosticar el origen del problema y tratar de resolverlo se encuentra en mi trabajo «Reason and Causation in Davidson's Theory of Action Explanation», Crítica, 30, 1998, pp. 29-43. 


\section{Emociones y acción}

Una pregunta que surge inmediatamente al considerar el modelo anterior de la explicación de la acción es cuál es el lugar que ocupan en él las emociones y los sentimientos. En contraste con la importancia que claramente parecen tener en la generación y explicación de muchas de nuestras acciones, los factores emocionales no aparecen explícitamente mencionados en dicho modelo. La razón de ello es que los proponentes del modelo no consideran necesaria una mención explícita de las emociones porque tienden a considerarlas como asimilables a deseos, creencias, o a una combinación de ambos.

En una primera aproximación, las emociones podrían ser incluidas en el componente desiderativo de las razones. Recordemos que, aunque el modelo anterior es frecuentemente conocido como el modelo creencia/deseo, Davidson habla en realidad, más cautamente, de «actitudes favorables», de las que el deseo sería sólo una forma, aunque seguramente la más representativa. Cabría quizá, con un poco de ingenio, analizar al menos algunas emociones como actitudes favorables hacia acciones que posean cierta propiedad (y, de nuevo, en la medida en que la posean). El miedo, por ejemplo, podría caracterizarse como una actitud favorable hacia (un deseo de llevar a cabo) acciones que tengan la propiedad de librarnos de un peligro, o de aquello que se teme; la ira, como una actitud favorable hacia acciones que produzcan un daño a la persona o cosa que es objeto de nuestra ira; y así sucesivamente. Estas actitudes, junto con las creencias correspondientes acerca de las acciones que tienen la propiedad en cuestión, constituirían razones para llevar a cabo tales acciones.

Sin embargo, la cuestión parece claramente más complicada, puesto que el miedo involucra también de algún modo la creencia en la existencia de un peligro, y la ira la creencia de que alguien nos ha causado un daño o ha intentado hacerlo. «Peligro» y «daño» son términos evaluativos. Las emociones, pues, involucrarían de algún modo creencias con un contenido al menos parcialmente evaluativo. El modo en cuestión podría ser el siguiente: esas creencias serían razones para las actitudes favorables correspondientes y, normalmente, darían lugar a ellas. Éstas, a su vez, en combinación con las creencias instrumentales acerca de las acciones adecuadas para satisfacer dichas actitudes desiderativas, serían razones para llevar a cabo tales acciones. Causadas del modo apropiado por tales razones, las acciones resultantes serían intencionales, en tanto que tendrían la explicación racional requerida por el modelo. Así, por ejemplo, la creencia de que algo es peligroso haría razonable y daría lugar al deseo de actuar con vistas a librarnos de ese algo, y este deseo, junto con la creencia de que (digamos) huir es el mejor modo de lograr ese objetivo, haría a su vez razonable la huida y daría lugar a ella. La huida causada por el miedo quedaría así integrada en esta concepción, sin necesidad de que el miedo mismo figure irreductiblemente en la explicación. 
En este marco, pues, las emociones serían actitudes desiderativas (generadas por creencias evaluativas) que constituirían uno de los componentes de una razón primaria, y, de este modo, quedarían integradas en el modelo creencia/deseo. No sería necesario añadirlas como elementos adicionales en la explicación racional de la acción intencional. Alternativamente, si las creencias evaluativas se consideran esenciales a las emociones, cabría concebir una emoción como un proceso que incluye una creencia (parcialmente) evaluativa que da lugar a una actitud desiderativa.

Se trata, pues, como vemos, de una concepción reductiva de las emociones. Según esta perspectiva, las emociones serían estados análogos a deseos, o bien combinaciones de tales estados con creencias (de contenido parcialmente evaluativo). Esta concepción presupone que las actitudes intencionales básicas son las creencias y los deseos, y que otras actitudes intencionales son analizables en términos de estas actitudes básicas. Es posible, sin embargo, rechazar este presupuesto y admitir una mayor variedad de actitudes intencionales básicas sin que ello afecte al núcleo esencial de esta concepción. Este núcleo esencial sería la consideración de las emociones como actitudes intencionales, como estados con un contenido intencional ${ }^{4}$. En este punto, la concepción de las emociones en el marco del modelo creencia/deseo enlaza con la llamada teoría cognitiva de las emociones que, en algunas de sus formas más moderadas, subraya el carácter esencial de la creencia en la emoción y, en sus formas más radicales, identifica las emociones con juicios o creencias evaluativas ${ }^{5}$. En realidad, desde un determinado punto de vista, la concepción de las emociones en el marco del modelo creencia/deseo puede concebirse como una versión particular de la teoría cognitiva, sobre todo si tenemos en cuenta que para Davidson un deseo o actitud favorable es una especie de juicio evaluativo: un juicio sobre la deseabilidad o bondad de acciones de cierto tipo.

Sin embargo, estas concepciones, que reducen las emociones a actitudes intencionales, dejan fuera de consideración aspectos que, aparentemente, son esenciales a aquéllas, en particular su peculiar fenomenología, su carácter de experiencias. No en vano las emociones son también denominadas «sentimientos», un término que destaca claramente su carácter de experiencias, de estados que son «sentidos» por un sujeto. Algo semejante cabría decir también de los cambios corporales característicos que acompañan a emociones como el miedo, la ira o el deseo sexual. ¿Puede hablarse realmente de miedo, ira o deseo sexual sin que estén presentes estos aspectos? La respuesta parece ser

${ }^{4}$ Sobre la base de esta concepción, la pregunta por la naturaleza última de las emociones no sería fundamentalmente distinta de la pregunta por la naturaleza última de las actitudes intencionales, y la respuesta a esta pregunta podría discurrir en términos diversos, dependiendo de las distintas concepciones metafísicas de tales actitudes: el conductismo, la teoría de la identidad, el funcionalismo, el monismo anómalo o el dualismo.

"Éste es el caso de Robert Solomon. Cf., por ejemplo, su artículo «Emotions and Choice», en A. O. Rorty (ed.), Explaining Emotions, Berkeley, University of California Press, 1982, pp. 252-281. 
claramente negativa. Pensemos que, en principio, parece perfectamente posible creer que algo es peligroso y considerar deseables acciones que permitan librarse del peligro sin sentir miedo en absoluto. Esto muestra que creencias y deseos no son suficientes para la emoción. Tampoco, sin embargo, parecen necesarios: los niños muy pequeños y los animales pueden tener determinadas emociones sin tener creencias y deseos con contenido proposicional. No parece, pues, correcta una perspectiva que reduce las emociones a actitudes intencionales como creencias y deseos. ¿Por qué, entonces, se ha llegado a sostener esta visión reductiva de la emoción? Para responder a esta pregunta hemos de atender, en primer lugar, a la génesis histórica del modelo creencia/deseo, con especial atención al concepto de deseo que se va configurando en este proceso.

\section{Interpretación, explicación y actitudes intencionales}

El referente más cercano de la concepción davidsoniana de la acción intencional es la obra de Elizabeth Anscombe, en particular su importante ensayo Intention ${ }^{6}$. En este ensayo encontramos ya, claramente expresada, la propuesta, desarrollada posteriormente por Davidson, de concebir la acción intencional en términos de la explicación mediante razones, así como la tesis según la cual una misma acción puede ser explicada por razones, y ser así intencional, bajo una descripción y no bajo otra, aun cuando ambas sean verdaderas. Davidson, que reconoce su deuda con Anscombe, aporta un análisis preciso del concepto de razón, en el sentido indicado más arriba, y de la explicación mediante razones. En este último aspecto, como vimos, Davidson subraya, por un lado, el modo en que la descripción de la acción bajo la cual ésta es intencional ha de estar relacionada con los contenidos de la creencia y el deseo del agente para que la explicación suministre el elemento de justificación racional que le es propio $\mathrm{y}$, por otro lado, insiste, frente a Anscombe, en que, además de esta relación entre el contenido de las razones y la descripción de la acción, ha de haber también una relación causal entre las razones y la acciones para que las primeras expliquen realmente las segundas.

El estudio de Anscombe se mueve claramente en el marco de la tradición wittgensteiniana, que opone de modo radical los conceptos de regla y razón, por un lado, y los de causa y ley, por otro. Sólo el primer par de conceptos es adecuado para el estudio y la explicación de la acción intencional humana, en contra de lo sostenido por el empirismo y el positivismo, que consideran las acciones sometidas a causas y leyes como cualesquiera otros fenómenos. Así, para empiristas y positivistas existe una continuidad entre las ciencias de la naturaleza y las ciencias sociales, en la medida en que ambas tienen

${ }^{6}$ G. E. M. Anscombe, Intention, 20. ${ }^{\mathrm{a}}$ ed., Oxford, Basil Blackwell, 1979 (10. ${ }^{\mathrm{a}}$ ed., 1957. Hay traducción castellana en Paidós/UAB). 
como tarea el descubrimiento de nexos causales y de leyes explicativas, mientras que la escuela wittgensteiniana defiende una esencial heterogeneidad entre uno y otro grupo de ciencias. Las ciencias sociales, a diferencia de las ciencias de la naturaleza, son esencialmente hermenéuticas o interpretativas. Su cometido, por decirlo en los términos del historicismo decimonónico, no es la explicación causal nomológica (Erklären), sino la comprensión (Verstehen).

Pensadores como Peter Winch proporcionaron una base precisa al concepto decimonónico de comprensión en el marco del análisis wittgensteiniano de las nociones de regla y razón ${ }^{7}$. La conducta específicamente humana es una conducta que sigue reglas, lo que significa que cabe distinguir en ella entre formas correctas e incorrectas de actuar y que se puede justificar y dar razones de lo que se hace por apelación a tales reglas. La justificación mediante la aportación de razones es el modo característico de dar cuenta de nuestras acciones, y este modo es sustancialmente distinto de la explicación y predicción de los fenómenos en términos de causas, regularidades y leyes. Una manera más concreta de expresar esta diferencia sería la siguiente: puesto que las reglas que se siguen son constitutivas de la acción que se lleva a cabo y puesto que la aportación de razones se produce en el marco de dichas reglas y por apelación a ellas, la justificación de la acción consiste en destacar relaciones de carácter interno entre el marco normativo de reglas, las razones y la acción, relaciones que presentan la acción como una conducta razonable (o al menos inteligible) a la luz de tales razones y reglas. Un corolario de ello es que la comprensión de las acciones humanas debe llevarse a cabo a partir de los conceptos y creencias de los propios agentes, puesto que son estos conceptos y creencias los que determinan lo que se está haciendo y por qué se está haciendo. Por el contrario, en la investigación causal lo que tratamos de establecer son relaciones entre el fenómeno que pretendemos explicar y otros fenómenos diversos y distintos como causas del mismo, o, por decirlo en términos de Hume, relaciones entre «existencias separadas», unidas entre sí únicamente como casos particulares de tipos de fenómenos entre los que se dan regularidades (leyes) de coexistencia y sucesión. Dicho de otro modo: la investigación causal establece relaciones externas entre fenómenos. Los conceptos que empleamos en esta clase de investigación no están constreñidos por conceptos o creencias presentes en los fenómenos investigados, sino que son impuestos desde fuera por el investigador en función de criterios como su fecundidad para el descubrimiento de leyes, la simplicidad o la capacidad explicativa. Aunque es razonable esperar, en el marco de una teoría explicativa general, que ciertos fenómenos tendrán lugar si se dan otros, los procesos causales mismos no son razonables o no razonables, a diferencia de las acciones humanas.

${ }^{7}$ P. Winch, The Idea of a Social Science, Londres, Routledge \& Kegan Paul, 1958 (hay traducción castellana en ed. Amorrortu). 
Advirtamos que en esta concepción de la tarea de las ciencias sociales los factores decisivos a tener en cuenta son los contenidos de las actitudes intencionales de los agentes cuyas acciones se pretende explicar, puesto que es el descubrimiento de relaciones entre tales contenidos, parcialmente determinados por el contexto social de instituciones y reglas, y su comportamiento lo que permite comprender este comportamiento como razonable y/o inteligible. En este marco, los estados emocionales de los agentes sólo resultan pertinentes en la medida en que posean un contenido que pueda relacionarse internamente con el de otros estados y con la acción. Las experiencias subjetivas que puedan acompañar o darse en tales estados, o en actitudes intencionales no emocionales, no contribuyen a la tarea de la comprensión, puesto que no son susceptibles de figurar como elementos de tales relaciones internas, de modo que carecen de interés para el teórico de la interpretación. Su papel en la generación de actitudes intencionales o de la acción, si lo tienen, es puramente causal y, por tanto, ajeno a la tarea de la comprensión.

En el marco del modelo creencia/deseo de la explicación de la acción, este discurso acerca de la comprensión como el descubrimiento de relaciones internas corresponde a la relación que se da en una razón primaria entre los contenidos de la creencia y del deseo del agente y la descripción de la acción bajo la cual ésta es intencional. Sólo a la luz de estas relaciones la acción aparece racionalmente justificada. La concepción davidsoniana de la acción intencional conecta, a través de Anscombe, con la tradición interpretativa wittgensteiniana y comparte con ella el desinterés por los aspectos fenomenológicos y experienciales de nuestra vida mental. Cabría pensar, sin embargo, que la presencia en Davidson del requisito causal, según el cual una razón sólo explica una acción si la causa, le aparta sustancialmente de esta tradición, acercándole a la concepción causal-explicativa de las ciencias sociales en el empirismo y el positivismo. Pero, en el marco de la filosofía davidsoniana, este requisito cumple más bien la función de conciliar su concepción de la acción intencional con una metafísica de carácter naturalista acerca del carácter en último término físico de las creencias y los deseos. No lleva a modificar la concepción de la tarea de las ciencias sociales en el sentido de la búsqueda de leyes y explicaciones causales. Por el contrario, para Davidson, como para Winch o Anscombe, las ciencias sociales son esencialmente interpretativas. La explicación de la acción intencional humana no es distinta de su interpretación, y tiende, en la terminología de Winch, al descubrimiento de relaciones internas entre razones y acción, no a la búsqueda de relaciones causales y de leyes. En realidad, para Davidson, esta búsqueda, que conecta las ciencias sociales con las ciencias de la naturaleza, está condenada al fracaso, porque el intento de explicar la acción humana está regida por el supuesto necesario del carácter básicamente racional de los agentes, que Davidson denomina el «principio constitutivo de la racionalidad», un supuesto que no desempeña papel alguno en las ciencias del mundo físico ${ }^{8}$. Así, para Davidson, las ciencias sociales

${ }^{8}$ Cf. su importante artículo «Mental Events», en Essays on Actions..., pp. 207-225. 
no están menos alejadas de las ciencias de la naturaleza que en la tradición interpetativa wittgensteiniana, a pesar de las diferencias que pueda haber en otros aspectos. La consideración de las emociones como actitudes intencionales y el desinterés por sus aspectos experienciales, por su carácter de sentimientos, así como por sus aspectos fisiológicos, es, en parte, una consecuencia de esta cercanía de Davidson a dicha tradición interpretativa.

\section{Cartesianismo y anticartesianismo}

En el marco de la tradición wittgensteiniana, la concepción interpretativa de las ciencias sociales, según la cual la tarea de comprender y explicar la acción humana consiste fundamentalmente en el descubrimiento de relaciones internas entre razones, reglas y comportamiento, no constituye una posición teórica autocontenida y aislada, sino más bien un corolario, que autores como Winch, Melden o Anscombe elaboran con detalle, de las investigaciones de Wittgenstein en los campos de la filosofía del lenguaje y la filosofía de la mente. En este último, los análisis de Wittgenstein apuntan hacia una visión global de la mente humana radicalmente opuesta a la no menos global concepción cartesiana, que en este aspecto no sólo incluye la filosofía del propio Descartes, sino también la tradición filosófica empirista y tendencias importantes del positivismo lógico. Problemas filosóficos clásicos, como el de la existencia del mundo externo o de otras mentes, derivan de esta concepción de la mente.

En la perspectiva cartesiana, los contenidos de la mente se caracterizan por su patencia consciente e inmediata a su portador. Ningún fenómeno o proceso físico exhibe esta característica. Como ha señalado Crispin Wright ${ }^{9}$, el cartesianismo concibe la totalidad de los estados mentales bajo el modelo de la sensación o la experiencia cualitativa subjetiva. La distinción actual entre estados intencionales y estados fenomenológicos no se encuentra realmente en pensadores como Descartes o Hume. Para éstos, una creencia o un deseo es un estado experiencial inmediatamente presente a la conciencia, como lo es una sensación. En el marco de esta perspectiva, la relación entre un estado mental y cierto comportamiento, dada la radical heterogeneidad entre ambos términos, sólo puede ser externa y, eventualmente, causal. Que un estado mental, concebido como un estado experiencial consciente, dé lugar a cierto comportamiento es una cuestión que sólo puede establecerse empíricamente. No hay nada en la naturaleza o concepto de la experiencia que incluya su relación constitutiva con un determinado comportamiento.

En sus investigaciones sobre conceptos mentales como el de comprensión o intención, Wittgenstein insiste, por el contrario, en que estos estados (y, en general, las actitudes intencionales) no tienen el carácter de experiencias

${ }^{9}$ Cf. C. Wright, «Wittgenstein's Later Philosophy of Mind: Sensation, Privacy, and Intention», Journal of Philosophy, 86, 1989, pp. 622-634. 
conscientes. A diferencia de una sensación de dolor, por ejemplo, estos estados no tienen, en expresión de Wittgenstein, «duración real» ${ }^{10}$. Las experiencias que puedan acompañar la comprensión o la intención son inesenciales a estos estados. Que un sujeto tenga una experiencia de «comprender» no establece que realmente comprenda. Y lo mismo sucede con la intención de hacer algo. La naturaleza del comprender o de la intención no es accesible a la conciencia o a la experiencia interna, sino que ha de investigarse atendiendo a la variedad de situaciones públicas en las que decimos de alguien que comprende o no, que tiene o no la intención de hacer algo, y al tipo de consideraciones o «criterios» que ponemos en juego para hacer estas afirmaciones. Y lo mismo cabe afirmar de otras actitudes intencionales. Si hacemos esto, veremos que es su comportamiento, o el modo en que desarrolla ciertas tareas, lo que determina nuestro juicio de que el sujeto comprende, tiene una intención determinada o desea hacer algo.

Un cartesiano, sin embargo, podría admitir que éste es, efectivamente, el modo en que atribuimos a otros sujetos estados como la intención, la comprensión o el deseo, pero insistiría en que, en la atribución de tales estados a nosotros mismos no atendemos en absoluto a nuestro comportamiento. Sabemos lo que deseamos o lo que tenemos intención de hacer de un modo inmediato y especialmente resistente al error. Y esto muestra, frente a cualesquiera apariencias en contra, que las intenciones o los deseos son estados de conciencia de los que tenemos experiencia inmediata. A diferencia de pensadores como Gilbert Ryle, Wittgenstein aceptó sin reservas que existía una asimetría entre el modo como atribuimos estados mentales a los demás y el modo como nos los atribuimos a nosotros mismos y que las autoatribuciones eran inmunes a muchas formas de error. Sin embargo, necesitaba dar cuenta de estos hechos sin comprometerse con la idea según la cual una autoatribución es un enunciado cognoscitivo, que expresa la conciencia inmediata que un sujeto tiene de sus propios estados mentales, puesto que admitir esta tesis significaba comprometerse con la concepción cartesiana de la mente y entrar en contradicción con su propio análisis de las actitudes intencionales. La propuesta que, sobre la base de un monto textual más bien exiguo, aunque significativo ${ }^{11}$, suele atribuirse a Wittgenstein consiste en que las autoatribuciones serían expresiones, no del conocimiento que un sujeto tiene de sus estados mentales, sino de los estados mentales mismos. Del mismo modo que el intento de alcanzar algo sería una expresión del deseo de conseguirlo, la emisión «deseo tal y tal» sería asimismo una expresión, en este caso verbal, del deseo de tal cosa, que sustituiría la expresión más primitiva, de carácter no verbal. La inmunidad al error de las autoatribuciones es, en el fondo, la misma que la inmunidad

\footnotetext{
${ }^{10}$ Wittgenstein escribe: «El propósito (la intención) no es ni una emoción, ni un estado de ánimo, ni una sensación o una imagen. No es ningún tipo de estado de conciencia. No tiene duración real» (Zettel, tr. de Octavio Castro y C. U. Moulines, México, UNAM, 1979, p. 45).

${ }^{11}$ Cf. especialmente el parágrafo 244 de las Investigaciones Filosóficas.
} 
al error de las expresiones no verbales de un estado mental: del mismo modo que no tiene sentido decir de un niño que trata de alcanzar una pelota que se equivoca, tampoco lo tiene decirlo de alguien que afirma sinceramente «deseo tal y tal». $\mathrm{Y}$ el hecho de que no atendamos a nuestro comportamiento cuando nos atribuimos, v. gr., un deseo no resulta sorprendente si pensamos que tampoco alguien que trata de alcanzar un objeto atiende a sus movimientos, sino al objeto que trata de alcanzar.

Esta propuesta, sea o no correctamente atribuible a Wittgenstein, ha sido generalmente aceptada por la escuela wittgensteiniana y constituye, históricamente, la primera gran alternativa plausible a la concepción cartesiana de las autoatribuciones de estados mentales. Aun cuando, a pesar de su atractivo inicial, tropiece en último término con dificultades tal vez insalvables, se trata sin duda de una propuesta interesante y digna de consideración ${ }^{12}$. En primer lugar, mantiene, también en la perspectiva de la primera persona, la relación interna entre los estados mentales y el comportamiento, en este caso expresivo, frente a la consideración cartesiana de tal relación como externa, y hace de un fenómeno público, como lo es el comportamiento expresivo, un elemento que constituye y determina la actitud intencional y su contenido ${ }^{13}$, frente a la separación cartesiana entre lo mental y cualquier fenómeno públicamente observable. En segundo lugar, da cuenta, al menos inicialmente, de los hechos anteriormente mencionados, relativos a la inmunidad al error y a la asimetría entre las atribuciones en primera y en tercera persona. Y, en tercer lugar, y sobre todo, honra una condición que cualquier teoría aceptable de las actitudes intencionales debe respetar, y que Wittgenstein aceptó sin duda a partir de las Observaciones filosóficas, a saber: que el contenido intencional de un deseo (o de otra actitud intencional) establece las condiciones de satisfacción del mismo (o de la actitud de que se trate) previamente al desarrollo contingente de los acontecimientos y con independencia de éste. Así, en las Observaciones Filosóficas, Wittgenstein escribe: «iAcaso mi teoría no se expresa totalmente en que el estado de cosas que satisface la esperanza de que $p$ se representa mediante la proposición $p$ ? No, por tanto, mediante la descripción de un acontecimiento completamente distinto» ${ }^{14}$. Suponer, en efecto, que lo que ahora deseo (o lo que creo) depende de lo que más adelante pueda contingentemente suceder conduce, como veremos, a consecuencias claramente absurdas. La con-

\footnotetext{
${ }^{12}$ Una importante crítica de la concepción expresiva de las autoatribuciones de estados mentales se contiene en C. Wright, «Self-Knowledge: the Wittgensteinian Legacy», en A. O'Hear (ed.), Current Issues in the Philosophy of Mind, Supplement to Philosophy, 43, 1998, pp. 101-122. Para una defensa reciente de la concepción expresiva de las autoatribuciones, con algunas modificaciones destinadas a afrontar objeciones, véase D. Bar-On y D. C. Long, «Avowals and First-Person Privilege», Philosophy and Phenomenological Research, 62, 2001, pp. 311-335. lógico.

${ }^{13}$ Lo que, por otra parte, compromete a Wittgenstein con una forma refinada de conductismo

${ }^{14}$ L. Wittgenstein, Philosophische Bemerkungen, Frankfurt, Suhrkamp, 1970 (10. a ed., 1964), parágrafo 25 .
} 
cepción wittgensteiniana respeta esta condición porque la expresión de un deseo, que fija su contenido, no depende de que el deseo expresado se satisfaga de hecho o no, o de otras circunstancias contingentes. El niño que trata de alcanzar la pelota expresa el deseo de alcanzarla con independencia de que finalmente acabe alcanzándola o abandone su intento por algún motivo.

Nos ocuparemos de esta importante cuestión con más detalle a continuación.

\section{El deseo y su objeto}

En su ensayo Free action, Abraham Melden, un integrante de lo que estamos llamando la tradición interpretativa wittgensteiniana, criticaba la concepción empirista del deseo como una «impresión de reflexión», una especie de tensión o ansiedad interna (es decir, en nuestros términos, como una forma de experiencia consciente) unida a cierta representación o idea ${ }^{15}$. Esta concepción del deseo no satisface la condición anteriormente indicada, puesto que hace depender el contenido del deseo (sus condiciones de satisfacción) de aquello que, contingentemente, acabe haciendo desaparecer esa tensión o ansiedad. En efecto, aun cuando el sujeto logre alcanzar el objeto representado en su mente, puede ocurrir que la tensión o ansiedad no desaparezca con ello, lo que mostraría que no era realmente ese objeto lo deseado. En cambio, podría ocurrir que otro objeto o suceso elimine esa ansiedad, lo que lo convertiría en el objeto o contenido del deseo. Una consecuencia de ello es que no podemos saber lo que deseamos en un momento dado sin esperar al desarrollo de los acontecimientos. Así, Melden escribe: «iCómo puedo decir, desde semejante concepción, qué es lo que deseo? No mediante la inspección del deseo mismo -la impresión interna, tensión, intranquilidad, prurito o crispación-, puesto que saber que deseo $y$ es tener cierto estado mental $x$ tal que $y$ disiparía dicho estado; y esto supone la posesión de un conocimiento causal que sólo puede fundarse en experiencias pasadas de que $y$ ha disipado $x$. Esto implica que la primera vez que deseé caviar no tendría ninguna razón en absoluto para suponer que era caviar, y no queso, lo que deseaba. Por todo lo que puedo decir sobre la base de la impresión o tensión del deseo mismo, podría muy bien suceder que el queso, o tal vez riñones crudos, en lugar del caviar, disipara esa impresión particular» ${ }^{16}$. Melden subraya, en un espíritu claramente wittgensteiniano, que lo esencial del deseo es su contenido intencional, y no cualquier experiencia que pueda acompañarlo, y que el deseo posee esencialmente ese contenido. Ninguna impresión o experiencia puede tener esa conexión esencial (interna) con un contenido u objeto que es propia del deseo: «El deseo es habitualmente identificado con algún suceso mental interno - un sentimiento de tensión o intranquilidad-. Sin embargo, como suceso interno, sea mental

\footnotetext{
${ }^{15}$ A. I. Melden, Free Action, Londres, Routledge \& Kegan Paul, 1961, cap. 10.

${ }^{16}$ Ibid., pp. 110-111.
} 
o fisiológico, ningún rasgo intrínseco del mismo revela su conexión con alguna otra cosa; pero como deseo, su misma caracterización involucra una referencia a la cosa deseada» ${ }^{17}$. Por lo tanto, un deseo no puede ser una impresión o experiencia interna ${ }^{18}$.

Wittgenstein puso de manifiesto las consecuencias absurdas de una teoría del contenido intencional que no satisfaga la condición anteriormente indicada al criticar la teoría del deseo que Bertrand Russell había expuesto en su obra Analysis of Mind ${ }^{19}$. De hecho, no es difícil percibir, en las observaciones de Melden sobre el deseo, los ecos de esta crítica de Wittgenstein a la teoría russelliana. Russell había sostenido que el contenido u objeto de un deseo era aquel suceso cuyo darse causaba el fin de un determinado ciclo de conducta. Russell pensaba en un caso como el siguiente: vemos a alguien yendo de una habitación a otra registrando cajones y armarios hasta que, de repente, saca un objeto del cajón que en ese momento estaba registrando, pone cara de satisfacción y deja de buscar. En una situación así, claramente diríamos que ése era el objeto que estaba buscando o que deseaba encontrar. En un famoso texto, Wittgenstein escribe contra Russell: «Creo que la teoría de Russell llevaría a lo siguiente: si doy a alguien una orden y lo que hace me produce alegría, entonces ha obedecido la orden. (Si yo quisiera comerme una manzana y alguien me diese un golpe en el estómago, de modo que mis deseos de comer desaparecieran, sería entonces ese golpe lo que deseaba)» ${ }^{20}$. $\mathrm{Y}$ en otros textos de la misma obra Wittgenstein reafirma la condición violada por la teoría del deseo de Russell ${ }^{21}$. Que un deseo se satisfaga o no es algo contingente, pero no es contingente que lo que se desea es una cosa y no otra. El deseo anticipa sus propias condiciones de satisfacción en su contenido (como la creencia anticipa en su contenido sus condiciones de verdad). Que esas condiciones se vean o no satisfechas no las determina (como no determina lo que uno cree el hecho de que la creencia resulte ser verdadera). Aun cuando la teoría de Russell tenga un sesgo conductista y la criticada por Melden sea más bien de tipo mentalista, ambas comparten unos mismos rasgos generales. Concretamente, ambas hacen depender las condiciones de satisfacción de un deseo

\footnotetext{
${ }^{17}$ Ibid., pp. 114-115.

${ }_{18}$ Sobre esta base, Melden subraya, frente a la perspectiva cartesiana, la conexión interna o «lógica» del deseo con la acción: nada puede ser un deseo en ausencia de un contenido, de algo que se desea, y cuando lo que se desea es llevar a cabo cierta acción, este deseo no puede ser la causa de que la acción se lleve a cabo, pues el contenido del deseo ya incluye el concepto de la acción, con lo que deseo y acción no son, en términos de Hume, «existencias separadas» y no pueden estar causalmente relacionados. Ésta es una forma del argumento de la «conexión lógica» en contra del carácter causal de las razones, que Davidson cuestionará en su defensa de dicho carácter.

${ }^{19}$ B. Russell, Analysis of Mind, Londres, Routledge \& Kegan Paul, 1921.

${ }^{20}$ Wittgenstein, Philosophische Bemerkungen, parágrafo 22.

${ }^{21}$ Así, en el parágrafo 29 leemos: «La descripción de la espera mediante lo que ella espera es una descripción interna.» $\mathrm{Y}$ en el parágrafo 34: «La espera de $p$ y el cumplimiento de $p$ corresponden a algo así como el molde y la forma interior de un cuerpo.»
} 
de contingencias empíricas, con lo que dichas condiciones (y con ellas el contenido intencional) quedan indeterminadas. Puesto que es algo contingente que un determinado suceso haga desaparecer mi ansiedad o me lleve a abandonar cierto comportamiento, una teoría del contenido intencional que haga depender este contenido de esas contingencias causales convierte también en contingente que un deseo tenga el contenido que tiene; pero esto no es contingente; es obviamente contingente que en un momento dado yo desee una cosa u otra, pero si deseo una cosa, no puedo desear algo distinto y seguir teniendo el mismo deseo. La relación entre el deseo (u otra actitud intencional) y su contenido es interna y constitutiva: dos deseos con contenidos distintos son deseos distintos (de distinto tipo). Las teorías empirista y russelliana convierten esa relación en externa y contingente, con las consecuencias absurdas indicadas por Wittgenstein y Melden.

Notemos también las consecuencias de tales teorías para la cuestión de las autoatribuciones de estados mentales y su especial inmunidad al error. Pensemos que con teorías de esa clase una autoatribución de un deseo pierde cualquier inmunidad al error y se convierte en una hipótesis empírica como cualquier otra, que será confirmada o rechazada por la experiencia. Pero las consecuencias de ello son devastadoras en este caso, ya que entonces nunca puedo estar seguro de lo que deseo (o lo que creo, o espero, etc.) y, por lo tanto, tampoco puedo estar seguro de si mi deseo ha sido satisfecho (o de si mi creencia es verdadera, o de si lo que espero ha sucedido, etc.), puesto que, para empezar, no puedo estar seguro de cuál es ese deseo (o esa creencia, o esa espera). Estimamos que estas consecuencias son claramente inadmisibles.

La contundente crítica de Wittgenstein a la teoría del deseo de Russell sumió a esta última en un descrédito del que ya no se recuperó. La concepción wittgensteiniana del deseo, y en general de las actitudes intencionales, que elimina de éstas cualquier elemento que haga depender el contenido o condiciones de satisfacción de una actitud de contingencias empíricas (futuras), fue aceptada sin reservas por la escuela wittgensteiniana y, a través de ella, y en especial de Anscombe, llegó a informar también la concepción davidsoniana de la mente y de la acción intencional y, en general, el modelo creencia/deseo de la explicación de la acción. Una consecuencia de ello fue el descrédito de los aspectos experienciales de nuestra vida mental, los sentimientos y las experiencias subjetivas, y su relegación a zonas marginales de la mente, dada la imposibilidad de integrar estos aspectos en el marco de relaciones internas que constituyen, desde esta perspectiva, los elementos esenciales en la comprensión de la acción humana. La reducción de las emociones a actitudes intencionales ha sido un corolario de todo ello.

Sin embargo, a pesar de la contundencia de la crítica de Wittgenstein, las teorías empirista y russelliana apuntan a cuestiones importantes para la comprensión de la naturaleza humana, que quedan fuera del alcance de lo que cabría llamar la perspectiva intencional de la tradición wittgensteiniana 
y del modelo creencia/deseo. Lo cierto es que sólo fragmentos limitados del transcurso de nuestra vida mental y nuestras acciones pueden ser cabalmente entendidos desde esa perspectiva. Aun cuando pueda haber relaciones de justificación racional entre nuestras creencias y deseos y nuestras acciones, muchas de tales creencias, deseos y acciones guardan también relaciones externas, puramente causales, con diversos factores, y son en ocasiones efectos brutos de fuerzas ajenas al reino de las razones y de las actitudes intencionales. Si bien muchos de nuestros deseos no se experimentan como tensión y ansiedad, sino que son, por decirlo así, puramente intencionales, hay deseos que se experimentan precisamente de ese modo. El deseo sexual hacia una determinada persona es quizá un ejemplo claro de ello. Por otra parte, experiencias subjetivas de tensión, ansiedad, tristeza o alegría hacen surgir en nosotros creencias y deseos de un modo puramente causal, ajeno a relaciones de justificación racional.

Permítasenos llamar la atención sobre un fenómeno bastante común, a saber, el hecho de que en ocasiones decimos «no era esto lo que yo deseaba» cuando es, por otro lado, evidente que, en algún sentido obvio, era eso precisamente lo que deseábamos. Pensemos, por ejemplo, en alguien que desea alcanzar una elevada posición social y que logra finalmente, tras muchos esfuerzos, satisfacer su deseo. Podemos entender perfectamente que esta persona, una vez satisfecho ese deseo, afirme sinceramente que no era eso lo que deseaba. No percibimos en ello ninguna contradicción, y podemos entender e incluso simpatizar con esa afirmación y con la persona que la hace. Esta persona no está diciendo que deseaba alcanzar una alta posición social y que no deseaba alcanzar una alta posición social. Sin embargo, si el contenido o condiciones de satisfacción del deseo se reducen a lo que sostiene la teoría de Wittgenstein o Davidson, a saber, a su contenido intencional, al hacer esa afirmación esta persona, o bien estaría mintiendo, o bien estaría incurriendo en una contradicción flagrante; y si era sincero y no se contradecía, sería necesario entonces poner en entredicho su autoridad sobre el contenido de sus deseos, de modo que, o bien antes el sujeto se equivocaba acerca de cuál era su deseo, o se equivoca ahora. Ninguna de las dos opciones resulta mínimamente satisfactoria.

Si suponemos, como parece plausible hacer, que la persona en cuestión es sincera, que no se está contradiciendo y que posee autoridad sobre lo que desea, un modo de dar cuenta de ello es que hay al menos dos sentidos distintos en que un deseo puede ser satisfecho y que, cuando el sujeto afirma que no ha alcanzado lo que deseaba, esto puede ser cierto en uno de los sentidos, y falso en el otro. En un reciente artículo, Ronald De Sousa ha distinguido precisamente, acerca del deseo, entre una satisfacción que él denomina semántica y una satisfacción emocional ${ }^{22}$. Por mi parte, propondría distinguir, en un sentido análogo, entre satisfacción intencional y satisfacción emocional, y entre contenido o condiciones de satisfacción intencional y contenido o con-

${ }^{22}$ R. De Sousa, «Desire and Serendipity», Midwest Studies in Philosophy, 22, 1998, pp. 120-134. 
diciones de satisfacción emocional. Las condiciones de satisfacción intencional de un deseo corresponderían a la concepción wittgensteiniana o davidsoniana del contenido intencional: si un sujeto desea que $p$, y $p$ es el caso, su deseo ha sido satisfecho en el sentido intencional del término. En este sentido, la afirmación de nuestro sujeto sería falsa. Pero él no está hablando desde este punto de vista. No está negando que ha alcanzado su deseo de lograr una alta posición social. Lo que está diciendo, seguramente, es que no se siente satisfecho emocional o anímicamente, que no ha logrado la felicidad o plenitud que esperaba. En el sentido de la satisfacción emocional de su deseo, la afirmación de nuestro sujeto, si es sincera, es presumiblemente verdadera: el sujeto no ha logrado su objetivo porque no se siente feliz, y nadie puede ser feliz si no se siente feliz.

La noción de satisfacción emocional conecta con la idea, criticada por Melden, según la cual un deseo es una experiencia de tensión o ansiedad interna, así como, un tanto más indirectamente, con la tesis de Russell según la cual el objeto de un deseo es aquello que pone fin a un ciclo de conducta. Esta noción de satisfacción emocional es, en nuestra opinión, un aspecto interesante y correcto de este tipo de teorías, un aspecto que quedó oscurecido por la devastadora crítica de Wittgenstein a la versión russelliana. Desde la perspectiva que hemos alcanzado, podemos decir que la crítica wittgensteiniana es correcta si interpretamos estas teorías como teorías del contenido o condiciones de satisfacción intencional del deseo. Hemos de reconocer que no ofrecen una concepción adecuada de dicho contenido. Pero la teoría wittgensteiniana, puramente intencional, del deseo fracasa a su vez por su incapacidad de integrar la noción de contenido o condiciones de satisfacción emocional.

Trataremos a continuación de profundizar en esta distinción.

\section{Satisfacción intencional y satisfacción emocional}

Señalemos, en primer lugar, que no existe una conexión necesaria entre la satisfacción intencional y la satisfacción emocional de nuestros deseos, como lo muestra claramente la posibilidad (y la realidad) de casos como el que hemos presentado más arriba. La discrepancia entre ambos tipos de satisfacción es siempre una posibilidad y, con bastante frecuencia, una realidad. La satisfacción emocional que esperamos alcanzar con la satisfacción intencional de nuestros deseos no está garantizada por ésta. Una manera de calibrar el grado de bienestar o malestar anímico de una sociedad puede ser precisamente analizar la frecuencia con la que la satisfacción emocional acompaña a la satisfacción intencional. No creemos que nuestras sociedades occidentales actuales alcancen un grado muy alto en esta supuesta escala, como lo sugiere la creciente incidencia de patologías emocionales entre nosotros, como la depresión o la ansiedad. 
Lo trágico de esta discrepancia potencial es que la satisfacción emocional, un concepto que no pretendemos muy alejado de lo que los antiguos griegos llamaron eudaimonia, es aquello a lo que en última instancia aspiramos («todos los seres humanos tienden por naturaleza a la felicidad», decía Aristóteles) y lo que nos lleva a procurar la satisfacción intencional de nuestros deseos, y, sin embargo, la satisfacción emocional está afectada por un grado de contingencia y de falta de control por nuestra parte mucho mayor que la satisfacción intencional. En realidad, la diferencia no es meramente de grado, sino de naturaleza. En el contexto de este trabajo podemos expresar esta diferencia diciendo que las condiciones de satisfacción emocional, a diferencia de las condiciones de satisfacción intencional, están regidas fundamentalmente por relaciones externas, en especial con la acción. Recordemos que la crítica de Wittgenstein a la teoría del deseo de Russell se basaba fundamentalmente en que esta última hacía depender el contenido del deseo de aquello que, contingentemente, viniera a dar fin a un ciclo de conducta, con lo que dicho contenido no estaría fijado previamente a tales contingencias. Esto hacía de la relación entre el deseo y su objeto una relación externa, con las consecuencias absurdas ya indicadas. Frente a esta concepción, Wittgenstein insistía en que el deseo contiene esencialmente su objeto, y anticipa en sí mismo aquel acontecimiento o acción que, de darse, lo satisfará. Así, mi deseo de que $p$ es aquel deseo que será satisfecho si, y sólo si, $p$. Esta observación es claramente adecuada como observación acerca del contenido intencional. Esta estructura de las actitudes intencionales permite que podamos saber por anticipado qué es lo que, de darse, satisfará nuestro deseo, es decir, cuáles son sus condiciones de satisfacción. Sin embargo, con respecto a las condiciones de satisfacción emocional de nuestros deseos, estamos en una situación mucho menos favorable. No sabemos por anticipado qué es lo que tendría que darse para que nuestro deseo se viese emocionalmente satisfecho. Asociamos a las condiciones de satisfacción intencional de nuestros deseos una imagen más o menos vaga de nuestra situación emocional si tales condiciones se vieran satisfechas, pero podemos comprobar dolorosamente que esa imagen era errónea. El deseo de vengarnos de una ofensa, que experimentamos, como diría Hume, como una tensión o ansiedad interna, nos lleva a asociar la venganza con la desaparición de esa tensión, pero puede ocurrir que la venganza, ya llevada a cabo, no produzca en nosotros la tranquilidad que esperábamos. Igualmente, el deseo sexual hacia una persona, que experimentamos también como tensión y ansiedad, puede verse satisfecho intencionalmente sin ser acompañado de la felicidad y ausencia de tensión que anticipábamos (omne animal triste post coitum, decían los latinos). Estamos, pues, con respecto a las condiciones de satisfacción emocional de nuestros deseos, en una situación muy cercana a la que se derivaba, según Wittgenstein, de la teoría russelliana del deseo: no sabemos cuáles son esas condiciones de satisfacción hasta que de hecho se satisfacen. Ésta es tal vez la base teórica que da cuenta de la indeterminación 
del contenido o condiciones de satisfacción de deseos pasionales como los indicados, un fenómeno sobre el que Amélie Rorty ha llamado la atención en un reciente trabajo: "La venganza es mía" puede imponer un coste a varias generaciones, porque las condiciones de su satisfacción son indeterminadas y abiertas, sin un límite bien definido. Y lo mismo sucede con otros deseos... pasionales. Así como no hay un término para la ira vengativa, no lo hay tampoco para la ambición, el honor, la gloria, ni para aquellos deseos que, como la codicia, la lujuria y la avaricia, son funcionalmente equivalentes a pasiones» ${ }^{23}$.

El deseo de venganza y el deseo sexual, así como los mencionados por Rorty, están ya muy próximos a las emociones. Podemos ahora pasar, desde la perspectiva que hemos alcanzado, a hablar directamente de ellas, con el fin de ofrecer algunas sugerencias sobre su lugar en la explicación e interpretación de la acción.

\section{Emociones y teoría de la acción}

La asimilación de las emociones a actitudes intencionales, y en particular a combinaciones de creencias y deseos, les asigna un contenido intencional, descuidando precisamente lo que estamos denominando contenido emocional. Por lo que respecta al contenido o condiciones de satisfacción propiamente emocional de las emociones, nuestras observaciones acerca de dichas condiciones en el caso de determinados deseos son también aplicables a las emociones y no vamos a volver sobre ellas. Es también cierto en este caso que no sabemos por anticipado cuáles son esas condiciones, qué tipo de circunstancias o experiencias producirá o restaurará (o, en el caso de estados emocionales positivos, mantendrá en nosotros) el equilibrio y bienestar emocional.

Pero la asimilación de las emociones a actitudes intencionales es incluso problemática en lo que respecta a la asignación misma de contenido intencional. Hay, en nuestra opinión, diferencias notables entre el contenido intencional de actitudes intencionales paradigmáticas, como la creencia, el deseo (no pasional) o la intención, y el contenido intencional de las emociones. En el caso de las primeras, cabe identificar plausiblemente, como hemos hecho hasta ahora, su contenido intencional con lo que genéricamente puede denominarse sus condiciones de satisfacción. Deseos, creencias e intenciones tienen condiciones de satisfacción que coinciden con su contenido, con lo que es deseado o creído, o con lo que se tiene intención de hacer. Lo que se desea (el contenido del deseo) es aquello que, de darse, satisfará el deseo. En el caso de la creencia, sus condiciones de satisfacción constituirían sus condiciones de verdad. Lo que se cree (el contenido de una creencia) es aquello que, de darse, haría

${ }^{23}$ A. O. Rorty, «Political Sources of Emotion», Midwest Studies in Philosophy, 22, 1998, pp. 21-33, p. 25. 
verdadera la creencia. En el caso de la intención, sus condiciones de satisfacción serían sus condiciones de cumplimiento. Lo que se pretende hacer (el contenido de la intención) es aquello que, de llevarse a cabo, daría cumplimiento a la intención. Pensemos ahora en casos paradigmáticos de emociones, como la ira, el miedo o la pasión amorosa. Es plausible pensar que estas emociones tienen contenido intencional en el sentido, más bien vago, de estar «dirigidas a» un objeto: aquello contra lo que se está airado, aquello que se teme o la persona a quien se ama. Advirtamos, en primer lugar, que, en algunos casos, este objeto no puede ser expresado, a diferencia del objeto de las actitudes intencionales paradigmáticas, en forma proposicional: en ocasiones, se está airado con una persona, o se teme a una persona o a una cosa, y, normalmente, se ama asimismo a una persona. Pero, en segundo lugar, no parece posible especificar el contenido de estas emociones del modo en que hemos especificado un poco más arriba el contenido de una creencia, un deseo o una intención, a saber, formulando sus condiciones de satisfacción. No podemos decir, siguiendo el modelo de las actitudes intencionales, que el contenido de la ira es aquello que, de darse, satisfaría la ira. Y lo mismo cabe decir del miedo o la pasión amorosa. En el caso del miedo o la ira, por ejemplo, si por «satisfacerse» entendemos «desaparecer», aquello que, de darse, hace desaparecer el miedo o la ira no es aquello que se teme o aquello con lo que se está airado. Por lo que respecta a la pasión amorosa, no podemos entender «satisfacerse» como «desaparecer», pues la desaparición de la pasión amorosa no equivale a su satisfacción. Tal vez por «satisfacción» hayamos de entender en este caso «consumación» o «unión con la persona amada», pero, una vez más, aquello que, en este sentido de «satisfacer», satisfaría la pasión amorosa no es su objeto, no es la persona amada. Así, pues, la conclusión parece ser que, aun cuando las emociones puedan tener un contenido u objeto intencional en el sentido vago de «ser acerca de» algo o «estar dirigidas a» algo, este contenido no es, en muchos casos, expresable en forma proposicional y, en segundo lugar, y posiblemente en todos los casos, no puede identificarse con sus condiciones de satisfacción. La razón de esto último es, seguramente, que las emociones no tienen propiamente condiciones de satisfacción intencional, al menos en el sentido bien definido en que las tienen las creencias, los deseos $\mathrm{o}$ las intenciones.

Las notables diferencias que el contenido intencional de las emociones presenta con respecto al de las creencias, deseos e intenciones tiene consecuencias importantes por lo que hace a la participación de las emociones en los procesos de razonamiento, incluido el razonamiento práctico que conduce a la acción. Así, que podamos emplear una creencia como base para inferir o deducir otras de ella depende esencialmente del hecho de que los contenidos de las creencias constituyen sus condiciones de verdad. Así, si la creencia de que $p$ se deduce de la creencia de que $q$, esto se debe a que las condiciones de verdad de $p$ están incluidas en las condiciones de verdad de $q$. Igualmente, 
si del deseo de que $p$ y de la creencia de que la acción $A$ dará lugar al hecho de que $p$ podemos inferir que la acción $A$ es deseable en la medida en que conduce a la satisfacción de nuestro deseo, ello se debe a que los contenidos de tales estados poseen condiciones de satisfacción (de verdad en el caso de las creencias). Así, las condiciones de verdad de la creencia anterior están internamente relacionadas con las condiciones de satisfacción del deseo, de modo que, si la creencia es verdadera, la acción $A$ conducirá al hecho de que $p$, lo que satisfará el deseo de que $p$. Sin embargo, si el contenido intencional de las emociones no puede entenderse en términos de condiciones de satisfacción, la participación de las emociones en la génesis de la acción habrá de tener un carácter distinto del de las creencias y deseos, siendo, presumiblemente, de tipo causal y externo más que racional e interno. Igualmente, la propia génesis de las emociones tendrá también este carácter, puesto que, no teniendo el tipo adecuado de contenido, no podrán inferirse de creencias, sino más bien ser causadas por ellas. Las emociones, pues, introducen en nuestra vida un elemento de contingencia y causalidad bruta que perturba la ordenada trama de relaciones internas que, en el modelo creencia/deseo, da origen y justifica nuestras acciones. Así, por ejemplo, la emoción de la ira, una vez surgida en nosotros, tiende a alimentarse a sí misma generando causalmente, y no racionalmente, creencias acerca del objeto de nuestra ira que lo presentan como más merecedor de la misma.

No se trata de negar que las emociones sean sensibles a nuestras creencias, como sostiene la concepción cognitiva. Sin duda, algunas lo son, como lo muestra el hecho de que, si abandonamos la creencia que causó en nosotros la ira hacia alguien, ésta tiende a extinguirse (repárese, en cambio, en cómo la pasión amorosa muestra en ocasiones una inercia notable frente al cambio de creencias acerca del amado o la amada). Pero, dado el carácter externo y causal de las relaciones recíprocas entre creencias y emociones, los resultados que un cambio en nuestras creencias (por ejemplo, a través de la terapia psicoanalítica) tiene en nuestros estados y personalidad emocionales son contingentes y frecuentemente imprevisibles (como bien lo saben los profesionales de ese tipo de terapia). En lo que respecta a la configuración y mejora, así como a la comprensión emocional de sí mismo o de otras personas, la sabiduría, en el sentido de tacto y experiencia, y de atención al caso particular por sí mismo, resulta absolutamente indispensable ${ }^{24}$.

Si las reflexiones que preceden son correctas, la asimilación de las emociones a actitudes intencionales estándar, en particular creencias y deseos, tropieza con dificultades muy importantes, que habrán de afectar a las diversas propuestas teóricas sobre la explicación de la acción que llevan a cabo esta asimilación, como el modelo creencia/deseo (y la tradición interpretativa wittgensteiniana del que deriva), la teoría de la decisión o la psicología cognitiva. Desde el punto de vista filosófico, la teoría de la acción habrá de tomar más

${ }^{24}$ Cf. al respecto mi trabajo «Emociones, racionalidad y responsabilidad», de próxima aparición. 
en serio la noción de causa y de relación externa en la conceptuación de la acción intencional y de su interpretación y explicación (en lugar de honrarlas sólo nominalmente, como de hecho hace la teoría davidsoniana). Hemos de estar preparados, al analizar la explicación de la acción humana, para emplear una trama de relaciones más compleja que la presupuesta por las propuestas mencionadas, admitiendo nexos causales brutos, ajenos a las relaciones de justificación racional entre contenidos intencionales, en distintos puntos del proceso que conduce a la acción. En una adecuada comprensión de estos nexos habrán de intervenir diversas teorías empíricas orientadas a la investigación causal y nomológica, incluyendo, además de la psicología empírica, las investigaciones neurofisiológicas y neuropsicológicas. Las reflexiones que preceden, realizadas desde un punto de vista puramente filosófico, son, sin embargo, congruentes con diversos resultados de estas últimas, que presentan los sistemas emocionales como más primitivos (ontogenética y filogenéticamente) que las funciones cognitivas superiores y parcialmente independientes de éstas ${ }^{25}$. Estas investigaciones empíricas, por su parte, pueden también obtener iluminación y ayuda de las reflexiones y análisis distintivamente filosóficos, que apenas podemos encontrar en aquéllas.

No obstante, sería erróneo pensar que el naturalismo científico es la conclusión general a extraer del presente trabajo. Tampoco, desde luego, el antinaturalismo. La conclusión es más bien que la complejidad de la acción y de la motivación humana requiere humildad y una aproximación epistemológica no menos compleja que aquéllas. La literatura, en particular, como ha mostrado recientemente Jon Elster ${ }^{26}$, es una valiosa fuente de conocimiento sobre estas cuestiones, y en particular sobre las emociones. Por otra parte, si, como hemos argumentado en este trabajo, algunos de nuestros deseos y todas nuestras emociones tienen condiciones de satisfacción emocional, y si el contenido intencional de las emociones es significativamente distinto del de las creencias, los deseos y las intenciones, en el sentido indicado más arriba, no podemos esperar una cabal comprensión y explicación de la acción humana sólo mediante el descubrimiento de relaciones internas de justificación racional entre contenidos intencionales de naturaleza proposicional que nos ofrece el modelo creencia/deseo o la tradición interpretativa wittgensteiniana. La comprensión de la acción humana requiere también la reivindicación de esa forma peculiar de cognición no proposicional que algunos pensadores decimonónicos denominaron Einfühlung: la empatía o la com-pasión. Es sintomático, y avala algunas de las tesis sostenidas en el presente trabajo, que, en su importante ensayo, ya mencionado anteriormente, Ciencia social y filosofía, Peter Winch sostenga que los defensores de estas formas de cognición malinterpretan la naturaleza

\footnotetext{
${ }^{25}$ Cf., en particular, J. Le Doux, The Emotional Brain. The Mysterious Underpinnings of Emotional Life, Nueva York, Simon \& Schuster, 1998 (10. ${ }^{\mathrm{a}}$ ed., 1996), esp. pp. 164-166.

${ }_{26} \mathrm{~J}$. Elster, Alchemies of the Mind. Rationality and the Emotions, Cambridge, Cambridge University Press, 1999.
} 
de la comprensión, y ofrezca de ellas una reducción a su propio concepto de comprensión, basado en las nociones de razón y regla. Los nexos brutos, puramente causales, vinculados a los estados emocionales y presentes en la génesis de la acción humana de los que hemos hablado no podrán ser cabalmente conceptuados sólo mediante investigaciones empírico-nomológicas, sino que requieren también la participación de nuestras capacidades empáticas y compasivas. En suma, el logro de un conocimiento más profundo y más complejo del complejo y profundo proceso que da lugar a nuestras intenciones y acciones no obedece sólo a un interés teórico, sino que responde y contribuye también a nuestra aspiración a la felicidad y al equilibrio anímico y emocional. 